\title{
UV-induction of keratinocyte endothelin-1 downregulates E-cadherin in melanocytes and melanoma cells
}

\author{
Sumayah Jamal ${ }^{1}$ and Robert J. Schneider ${ }^{2}$ \\ ${ }^{1}$ The Ronald O. Perelman Department of Dermatology, and \\ ${ }^{2}$ The Department of Microbiology, New York University School of Medicine, New York, New York, USA
}

\begin{abstract}
Endothelin-1 (ET-1), a peptide that is secreted by keratinocytes in the skin in response to ultraviolet irradiation, is a ligand for the endothelin-B $\left(\mathrm{ET}_{\mathrm{B}}\right)$ receptor. Blockade of this receptor inhibits melanoma cell growth and induces cell death in vivo and in vitro. Additionally, $\mathrm{ET}_{\mathrm{B}}$ is a melanoma progression marker. These findings suggest that the $\mathrm{ET}-1 / \mathrm{ET}_{\mathrm{B}}$ receptor pathway contributes to melanoma development or progression. Here, we demonstrate that activation of the ET-1/ET $\mathrm{B}$ pathway downregulates E-cadherin and associated catenin proteins in human melanocytes and melanoma cells. E-cadherin is an established suppressor of melanoma cell invasion in vitro and in vivo. Downregulation of $\mathrm{E}$-cadherin by $\mathrm{ET}-1 / \mathrm{ET}_{\mathrm{B}}$ involves the downstream activation of caspase- 8 but not of distal, executioner caspases, and does not lead to apoptosis. ET-1 also induces a transient association between caspase- 8 and E-cadherin: $\beta$-catenin complexes. Hence, activation of the ET- $1 / \mathrm{ET}_{\mathrm{B}}$ pathway promotes molecular events known to promote melanoma invasion.
\end{abstract}

J. Clin. Invest. 110:443-452 (2002). doi:10.1172/JCI200213729.

\section{Introduction}

Melanocytes are the pigment-producing cells of the epidermis that undergo malignant transformation in malignant melanoma. Through their dendritic processes, melanocytes contact multiple keratinocytes, the predominant cell type in the epidermis. The adhesion molecule E-cadherin mediates cell:cell contact between these two cell types (1). Keratinocytes control the growth rate and phenotype of melanocytes in the epidermis in an E-cadherin-dependent fashion (2). In vitro, loss of contact with keratinocytes causes melanocytes to dedifferentiate and to express melanoma-associated adhesion molecules (3). E-cadherin, which is a tumor invasion suppressor, is downregulated in most melanoma cells, and this renders them refractory to keratinocyte-mediated control (2). Forced expression of E-cadherin in these cells restores keratinocyte-mediated control and inhibits melanoma invasion in vitro and in vivo (2). Since mutations of E-cadherin have not been discovered in melanoma cells, an epigenetic cause for the decrease in E-cadherin expression levels has been postulated (2).

Endothelin-1 (ET-1) is a 21-amino acid peptide secreted by keratinocytes, which stimulates proliferation, chemotaxis, and pigment production in

Received for publication July 10, 2001, and accepted in revised form June 10, 2002.

Address correspondence to: Sumayah Jamal, The Ronald O. Perelman Department of Dermatology, 550 First Avenue, New York University School of Medicine, New York, New York 10016, USA. Phone: (212) 263-3863; Fax: (212) 263-8276; E-mail: jamals01@popmail.med.nyu.edu.

Conflict of interest: No conflict of interest has been declared. Nonstandard abbreviations used: endothelin-1 (ET-1); ultraviolet irradiation (UVR); endothelin- $\mathrm{B}\left(\mathrm{ET}_{\mathrm{B}}\right)$; melanoma growth medium (MGM); 12-O-tetradecanoyl phorbol-13-acetate (PMA); horseradish peroxidase (HRP); inhibitor of apoptosis protein (IAP). melanocytic cells $(4,5)$. Ultraviolet irradiation (UVR), which is strongly linked to melanoma development, induces a marked and sustained increase in ET-1 secretion by keratinocytes $(6,7)$. A recent report demonstrates focal epidermal ET-1 hypersecretion and endothelin- $\mathrm{B}\left(\mathrm{ET}_{\mathrm{B}}\right)$ overexpression in human skin samples from pigmented skin lesions called lentigo senilis (8). $\mathrm{ET}_{\mathrm{B}}$ receptor expression gradually increases as melanocytic lesions progress from nevi to malignant melanomas, suggesting that the $\mathrm{ET}_{\mathrm{B}}$ receptor, as well as its ligand, ET-1, play a role in the tumor progression of malignant melanoma (9). Another report has demonstrated that $\mathrm{ET}_{\mathrm{B}}$ receptor blockade induces growth arrest and death of melanoma cells in vivo and in vitro (10). All of these studies suggest an important role for the ET-1/ET $\mathrm{B}_{\mathrm{B}}$ receptor pathway in the development of melanocytic neoplasms, but the molecular basis for this role is unknown. Here, we demonstrate that ET-1 is a potent downregulator of E-cadherin in human melanocytes and melanoma cells. We describe a novel mechanism for this response, which involves activation of the $\mathrm{ET}_{\mathrm{B}}$ receptor and activation of caspase-8. We further demonstrate that low levels of ET-1 secreted by keratinocytes in response to UVR are sufficient to downregulate E-cadherin in melanocytic cells.

\section{Methods}

Cells and cell culture. Uli Rodeck (Thomas Jefferson University, Philadelphia, Pennsylvania, USA) kindly donated the FM2030 and FM2050 cells. CL-NHEM and CASNHEM melanocytes were purchased from Clonetics Corp. (San Diego, California, USA) and Cascade Biologics (Portland, Oregon, USA), respectively. The 976 and 982 cells were kindly donated by Zalfa Abdel-Malek (University of Cincinnati, Cincinnati, Ohio, USA). The 71201-L, 8101D-2, and 90701-D cells were isolated 
from neonatal foreskins as described (11). SKMEL28 and M20 cells were a generous gift from J.C. Bystryn (New York University School of Medicine, New York, New York, USA). A375, WM-266-4, and WM-115 melanoma cells were purchased from the American Type Culture Collection (ATCC; Rockville, Maryland, USA). WM123 cells were a gift from Meenhard Herlyn (The Wistar Institute, Philadelphia, Pennsylvania, USA). Human epidermal keratinocytes and culture medium (EPI-life) were purchased from Cascade Biologics. Culture conditions for melanocytes and melanoma cells were as described (2). All melanocytes used were from passage 5 to 15 , and all were from normal nontransformed primary cell culture isolates. Briefly, melanoma growth medium (MGM) was four parts MCDB153 (Sigma-Aldrich, St. Louis, Missouri, USA), one part L15, insulin $5 \mu \mathrm{g} / \mathrm{ml}$, transferrin $10 \mu \mathrm{g} / \mathrm{ml}, 4 \%$ FBS (HyClone Laboratories, Logan, Utah, USA), and $1 \mathrm{mM} \mathrm{Ca}^{2+}$. FBS was reduced to $2 \%$ for ET- 1 stimulations. Melanocyte growth medium was MGM supplemented with $10^{-7} \mathrm{M}$ 12-O-tetradecanoyl phorbol-13-acetate (PMA), 80 $\mu \mathrm{g} / \mathrm{ml}$ bovine pituitary extract, $5 \mathrm{ng} / \mathrm{ml}$ human EGF. Two weeks prior to ET stimulation, water-soluble 12,13phorbol dibutyrate $\left(10^{-6} \mathrm{M}\right)$ was substituted for TPA. Before ET stimulation, melanocytes were grown for 2 days in phorbol-free melanocyte growth medium. All media and supplements were obtained from Invitrogen Life Technologies Inc. (Carlsbad, California, USA) unless otherwise noted. Where indicated, $1 \mu \mathrm{M}$ BQ123 and $1 \mu \mathrm{M}$ BQ788 were added to cells 1 hour before ET-1 addition. ET-1, ET-3, BQ123, BQ788, were from Peninsula Laboratories Inc. (Belmont, California, USA). Caspase inhibitors (218773, 235423, 218767,400011, 218776; Calbiochem-Novabiochem Corp., San Diego, California, USA) all contained a 16 -amino acid signal peptide derived from Kaposi fibroblast growth factor to confer cell permeability, followed by the specific 4-amino acid caspase cleavage site (Ac-AAVALLPAVLLAALAPXXX'D-CHO).

Western blot analysis. For E-cadherin analysis, cells were lysed in RIPA buffer (1\% NP-40, 0.5\% deoxycholic acid, $10 \mathrm{mM}$ Tris, pH 8.0, $150 \mathrm{mM} \mathrm{NaCl}, 50 \mathrm{mM} \mathrm{NaF}, 0.2$ $\mathrm{mM}$ sodium vanadate, $1 \mathrm{mM}$ DTT, $1 \times$ protease inhibitors-Boehringer complete; Roche, Indianapolis, Indiana, USA). Lysates were quantitated using protein assay reagent (Bio-Rad Laboratories, Hercules, California, USA) and $1.0 \mu \mathrm{g}$ of protein per sample used for $7 \%$ SDS-PAGE (unless samples were prepared from A375, WM-266-4, or WM-115 cells, in which case $100 \mu \mathrm{g}$ of protein per sample was used). For analysis of $\beta$-catenin and $\mathrm{p} 120^{\mathrm{CTN}}$, membrane-enriched fractions were prepared as follows: cell pellets were resuspended in threepellet volumes of buffer A (10 mM HEPES, $\mathrm{pH} 7.5,1.5$ $\mathrm{mM} \mathrm{MgCl}, 10 \mathrm{mM} \mathrm{KCl}, 10 \mathrm{mM} \mathrm{NaF}, 1 \mathrm{mM}$ sodium vanadate, $2 \mathrm{mM}$ EDTA, $1 \times$ protease inhibitorsBoehringer complete/EDTA-free, $1 \mathrm{mM}$ DTT) and allowed to swell for 10 minutes on ice. Cell membranes were disrupted by vortexing for 20 seconds, and membranes were pelleted by centrifugation for 10 seconds.
Supernatants containing cytoplasmic proteins were discarded. Membrane pellets were then solubilized in RIPA buffer as described above. Samples containing 2.5 $\mu \mathrm{g}\left(\mathrm{p} 120^{\mathrm{CTN}}\right)$ or $0.6 \mu \mathrm{g}$ ( $\beta$-catenin) of protein were analyzed by $7 \%$ SDS-PAGE. For analysis of caspases, crude cytoplasmic extracts were prepared as described for the catenin proteins, except that cytoplasmic supernatants were supplemented with 0.1 vol of buffer B $(0.3 \mathrm{M}$ HEPES, pH 7.5, $0.03 \mathrm{M} \mathrm{MgCl}_{2}, 1.4 \mathrm{M} \mathrm{KCl}$ ). Supernatants were then clarified by centrifugation, and $20 \mu \mathrm{g}$ of protein per sample were analyzed by $14 \%$ SDS-PAGE for caspase immunoblots. All except caspase blots were developed using the enhanced chemiluminescence detection system (Amersham Biosciences, Piscataway, New Jersey, USA). Caspase blots were developed using Super Signal West Femto Maximum Sensitivity Substrate (Pierce Chemical Co., Rockford, Illinois, USA). Ab's were as follows: anti-E-cadherin, anti-pp120, anti- $\beta$-catenin, anti-caspase- 3 , -7 (Transduction Laboratories, Lexington, Kentucky, USA); anti-CD44, anti-ICAM-1 (R\&D Systems Inc., Minneapolis, Minnesota, USA); anti-N-cadherin (a gift from Peggy Wheelock, University of Idaho, Moscow, Idaho, USA); anti-E-cadherin-NT (anti-E-cad-NT) (clone HECD-1; Zymed Laboratories Inc., San Francisco, California, USA); anti-mouse and anti-goat IgG horseradish peroxidase (HRP; Santa-Cruz Biotechnology Inc., Santa Cruz, California, USA), anti-caspase-8 antibody (Upstate Cell Signaling Solutions, Waltham, Massachusetts, USA). The anti-caspase-8 antibody was used at a 1:4,000 dilution in TBST ( $10 \mathrm{mM}$ Tris, $\mathrm{pH} 8.0,150$ $\mathrm{mM} \mathrm{NaCl}, 1 \%$ Tween 20 ), filtered through a $0.2-\mu \mathrm{M}$ filter, and then supplemented with $1 \%$ powdered milk. Anti-mouse IgG HRP was then used at a 1:5,000 dilution, then filtered and supplemented with powdered milk as above. All caspase Ab's used were directed against epitopes within the caspase catalytic domains and therefore recognized both the zymogen and catalytic subunits of the capases in question.

Northern blot analysis. Total RNA was isolated from cells using TRIzol reagent (Invitrogen Life Technologies Inc.). RNA ( $20 \mu \mathrm{g}$ per sample) was subjected to agarose gel electrophoresis, and samples were transferred to nylon membranes. E-cadherin, $\beta$-catenin, and GAPDH-radiolabeled cDNA probes were generated using RediPrime (Amersham Biosciences).

Ultraviolet B irradiation of keratinocytes, IL-1 stimulations, and ET-1 ELISA. Ultraviolet B (UVB) irradiation was carried out using a Stratagene 2000 illuminator specially equipped with FG15T8 bulbs, which produce maximal output in the UVB range. Irradiations were carried out per established protocols as described previously $(6,12)$. These protocols report the need for multiple rounds of irradiation spaced at 48-hour intervals to induce keratinocyte secretion of ET-1. We first titrated the UVB dose to the percentage of cell viability and determined that doses greater than $2 \mathrm{~mJ} / \mathrm{cm}^{2}$ resulted in greater than $5 \%$ cell death; therefore, we used doses of less than or equal to 2 $\mathrm{mJ}$. Briefly, keratinocytes at $90 \%$ confluence in $10-\mathrm{cm}^{2}$ 
dishes were rinsed once in PBS, which was then aspirated, and cells were irradiated without tissue culture dish lids. Cells were then fed $5 \mathrm{ml}$ of fresh medium. Two subsequent irradiations were performed at 48-hour intervals with replacement of original medium afterward. Medium was supplemented with $2 \mathrm{ml}$ of fresh medium after the last irradiation, and conditioned medium was then collected 24 hours later. IL-1-keratinocyte-conditioned medium (KCM) was collected after a 4-day stimulation of keratinocytes with $40 \mathrm{pg} / \mathrm{ml}$ purified recombinant human IL-1 $\alpha$ (Calbiochem-Novabiochem Corp.). ET-1 concentrations in $\mathrm{KCM}$ were determined using a colorimetric ELISA kit for human ET-1 (R \& D Systems Inc.).

Immunoprecipitation. Twenty micrograms of membrane-enriched fractions (prepared as described above) were diluted in $500 \mu \mathrm{l}$ of IP buffer (10 mM Tris, $\mathrm{pH}$ 8.0,
$150 \mathrm{mM} \mathrm{NaCl}, 0.5 \% \mathrm{NP}-40$, protease inhibitors), precleared with $25 \mu \mathrm{l}$ anti-mouse IgG agarose (SigmaAldrich), then incubated with $5 \mu \mathrm{g}$ anti-caspase- $8 \mathrm{Ab}$ (Upstate Cell Signaling Solutions) for 1 hour on ice. After addition and incubation with $25 \mu$ lof anti-mouse IgG agarose, complexes were washed twice in IP buffer and boiled in $50 \mu \mathrm{l} 2 \times$ SDS-PAGE loading buffer. Ten microliters per sample was used for E-cadherin and $\beta$-catenin immunoblots, and $25 \mu \mathrm{l}$ was used for $\mathrm{p} 120^{\mathrm{CTN}}$ immunoblots. For the $\beta$-catenin immunoprecipitations, lysates were first normalized for $\beta$-catenin content as determined by Western blot analysis. The starting amount of protein ranged from 5 to $25 \mu \mathrm{g}$. Immunoprecipitations were then carried out as described above using $5 \mu \mathrm{g}$ of anti- $\beta$-catenin $\mathrm{Ab}$. Twenty-five microliters of sample was used for E-cadherin Western blot analysis.
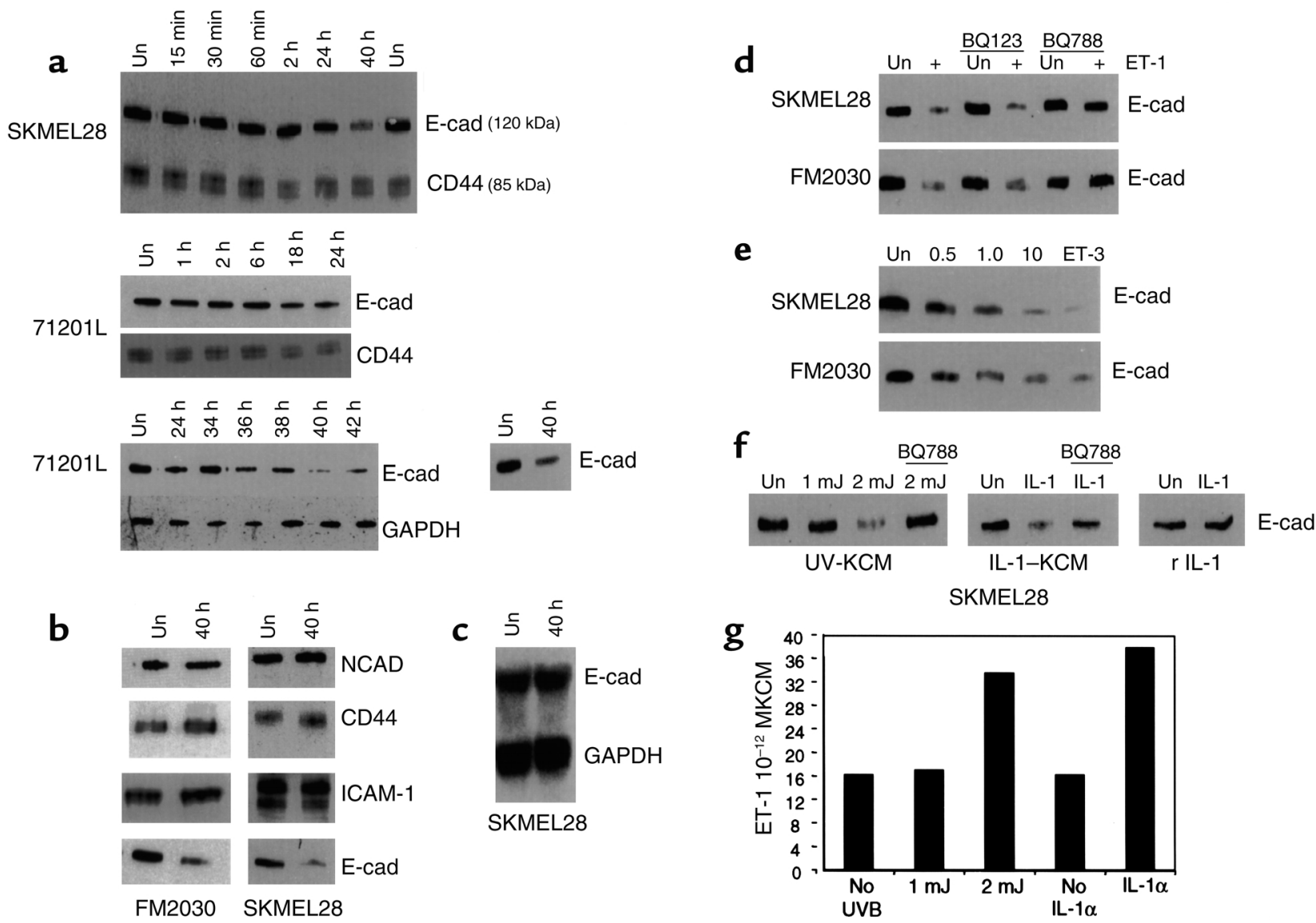

Figure 1

Downregulation of E-cadherin by ET-1 in melanoma cells (SKMEL28) and melanocytes (71201L, FM2030). Lysates normalized for protein content were analyzed for E-cadherin protein levels by immunoblot analysis. (a) Cells were stimulated with $10 \mathrm{nM}$ ET-1 for the indicated times. Un, unstimulated controls. ET-1 was removed after 3 hours, culture medium replaced with ET-1-free medium, and cells harvested at 40 hours (lower-right panel). (b) N-cadherin, CD44, and ICAM-1 are not affected by ET-1. (c) E-cadherin Northern blot of total RNA isolated from SKMEL28 cells. GAPDH serves as an internal control. Identical results were obtained using melanocytes (data not shown). (d) $\mathrm{ET}_{\mathrm{B}}$ receptor antagonist $\mathrm{BQ788}$ blocks E-cadherin downregulation by $\mathrm{ET}-1$. Cells were incubated with either $\mathrm{ET}_{\mathrm{A}}$ receptor antagonist $\mathrm{BQ} 123$ or $\mathrm{ET}_{\mathrm{B}}$ receptor antagonist $\mathrm{BQ788}$ as indicated. (e) E-cadherin downregulation by ET-1 is dose responsive. Lanes 2-4 are labeled with the

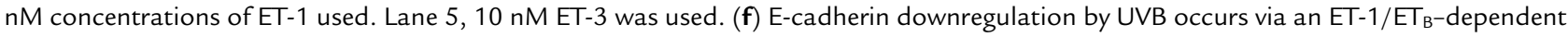
pathway. Conditioned medium from UV-irradiated keratinocytes (UV-KCM) was used to stimulate SKMEL28 cells for 40 hours (left panel). First lane, KCM from unirradiated control (Un). Last lane, cells were pretreated with $\mathrm{ET}_{\mathrm{B}}$ receptor antagonist BQ788. Cells were stimulated for 40 hours with conditioned medium from keratinocytes stimulated with IL-1 $\alpha$ (middle panel). Cells were stimulated with purified recombinant purified IL-1 $\alpha$ (right panel). Identical results were obtained using melanocytes (data not shown). (g) ET-1 ELISA assay of UV-KCM and IL-1-KCM. Results shown are representative of at least four independent experiments. E-cad, E-cadherin. 
Table 1

Downregulation of E-cadherin by ET-1 in primary melanocyte cultures and melanoma cell lines

$\begin{array}{ll}\text { Human primary melanocytes } & \text { Human melanoma cell lines } \\ \text { FM2030 } & \text { SKMEL28 } \\ \text { FM2050 } & \text { M20 } \\ \text { FM72 } & \text { WM-266-4 } \\ 976 & \text { WM123 } \\ 982 & \text { WM-115 } \\ \text { CL-NHEM } & \text { A375 } \\ \text { CAS-NHEM } & \\ \text { 8101D-2 } & \\ \text { 71201-L } & \\ \text { 90701-D } & \\ \text { A E-cadherin undetectable at baseline. } & \end{array}$

Stimulation of cells with ET-1-conditioned medium. SKMEL28 cells $\left(10^{6}\right)$ in $10-\mathrm{cm}^{2}$ dishes were stimulated with 10 nM ET-1. After 40 hours, conditioned medium was collected from dishes as well as from unstimulated control dishes. Conditioned medium $(4 \mathrm{ml})$ was added to SKMEL28 cells, or the melanocytes were seeded at $50 \%$ confluence in $60-\mathrm{cm}^{2}$ dishes. Cells were monitored at the indicated time points and examined for E-cadherin protein levels as described previously.

\section{Results}

ET-1 downregulates E-cadherin. ET-1 typically elicits delayed intracellular signaling responses in melanocytes. ET-1-dependent dendrite extension in melanocytes occurs maximally at 48 hours after stimulation (12). In addition, ET-1 induced-fibroblast-like morphology changes in melanocytes are best appreciated at time points beyond 48 hours (11). Delayed and sustained responses to ET-1 are also commonly observed in vascular smooth muscle cells (13). Thus, a delayed physiologic response to ET-1 stimulation is commonly observed. Accordingly, we investigated the effect of ET-1 on E-cadherin protein levels at both early and late time points. Human neonatal melanocytes (71201L and FM2030) and human melanoma cells (SKMEL28) were stimulated with synthetic purified ET-1 at a $10-n M$ concentration over a 40 -hour time course, and lysates were examined for E-cadherin protein levels. This concentration of ET-1 is identical to that used in most reports. At early time points, spanning 15 minutes to 24 hours after ET- 1 stimulation, no significant change in E-cadherin protein levels was detected in either cell type (Figure 1a, upper-middle and lower-left panels). However, by 40 hours, an approximate tenfold decrease in E-cadherin protein is evident in both cell types. Stimulation of cells with ET-1 for just 3 hours, followed by its withdrawal, still resulted in a delayed downregulation of E-cadherin observed at the 40-hour time point (Figure 1a, lower-right panel). This suggests that the critical ET-1-mediated signaling events occur within the first 3 hours of ET- 1 stimulation and that the subsequent presence of ET- 1 is not necessary for E-cadherin downregulation to occur. It should be noted that the delayed kinetics of the response are seen in both melanocytes and melanoma cells despite the fact that only melanocyte growth medium, unlike MGM, contains phorbol ester. This suggests that the kinetics represent a phenomenon that is not influenced by phorbol-responsive pathways. The downregulation of E-cadherin by ET-1 was observed in eight other primary melanocyte cultures and three additional melanoma cell lines (M20, WM123, and WM-266-4; Table 1). In A375 and WM-115 melanoma cells, E-cadherin protein was undetectable at baseline. It is unlikely that the decrease in E-cadherin protein levels observed in ET-1-treated cells is due to ET-1-dependent mitogenesis because such a mechanism would require a tenfold increase in cell number between 24 and 40 hours, and neither melanocytes nor melanoma cells divide this rapidly. In addition, one would expect to see a similar decrease in other cell surface molecules. As demonstrated, ET-1 had no effect upon expression levels of three such molecules: CD44, N-cadherin, and ICAM (Figure 1, $\mathrm{a}$ and $\mathrm{b}$ ). These results also demonstrate the specificity of the response for E-cadherin. Northern blot analysis of E-cadherin mRNA demonstrated that its abundance was unchanged by treatment with ET-1, suggesting a posttranscriptional mechanism of downregulation (Figure 1c). Thus, ET-1 downregulated E-cadherin with the delayed kinetics characteristically observed for other ET-1 effects.

Downregulation of E-cadherin by ET-1 is dose responsive and requires activation of the $E T_{B}$ receptor. There are two well-characterized ET-1 receptors, an A subtype $\left(\mathrm{ET}_{\mathrm{A}}\right)$ and a $B$ subtype $\left(\mathrm{ET}_{\mathrm{B}}\right)$ (14). Both subtypes are expressed in melanocytes, while most melanoma cells, including SKMEL28 cells, lose expression of the $\mathrm{ET}_{\mathrm{A}}$ and express only the $\mathrm{ET}_{\mathrm{B}}$ receptor (15). ET-1 binds with equal affinity to both receptor subtypes $(4,5,16)$. To determine which receptor subtype mediates E-cadherin downregulation, studies were repeated in the presence of a selective $\mathrm{ET}_{\mathrm{A}}$ receptor antagonist, $\mathrm{BQ} 123$ (4), or a selective $\mathrm{ET}_{B}$ receptor antagonist, BQ788 (10). Both antagonists prevent ET-1 receptor binding and were used at a 100-fold molar excess relative to ET-1, a concentration widely shown to effectively block ET-1 binding (17). BQ788, but not BQ123, blocked E-cadherin downregulation by ET-1 in both melanocytes and melanoma cells (Figure 1d), indicating that $\mathrm{ET}_{B}$ receptor activation is required for this response. In addition, SKMEL 28 cells do not express the $\mathrm{ET}_{\mathrm{A}}$ receptor (15), further reinforcing that the response is mediated by $\mathrm{ET}_{\mathrm{B}}$. Downregulation of E-cadherin by ET-1 is dose-responsive in both melanocytes and melanoma cells (Figure 1e, lanes 1-4). ET-3, a selective $\mathrm{ET}_{\mathrm{B}}$ receptor agonist that is not secreted by keratinocytes (14), also potently downregulated E-cadherin (Figure 1e, far right lanes), providing a second line of evidence that the $\mathrm{ET}_{\mathrm{B}}$ receptor mediates the response. Since keratinocytes do not secrete ET-3 (6), all further studies were performed using ET-1. 
These results reveal a previously unknown function for $\mathrm{ET}_{\mathrm{B}}$. Mutation of the $E T_{B}$ receptor causes migratory failure of neural crest-derived cell populations during embryogenesis, resulting in Waardenburg-Shah syndrome in humans (patchy absence of epidermal melanocytes and aganglionic megacolon) (14). During the migratory phase of neural crest cells, suppression of E-cadherin expression through $\mathrm{ET}_{\mathrm{B}}$ receptor activation likely prevents premature cell aggregation. Most melanoma cells do not express the $\mathrm{ET}_{\mathrm{A}}$ receptor (15), making them phenotypically similar to undifferentiated melanocyte precursors that express the $\mathrm{ET}_{\mathrm{B}}$ and lack the $\mathrm{ET}_{\mathrm{A}}$ receptor (18). In the adult, loss of the $\mathrm{ET}_{\mathrm{A}}$ receptor in the melanoma cell, coupled with overexpression of the $\mathrm{ET}_{\mathrm{B}}$ receptor and epidermal ET-1 hypersecretion, may recapitulate the embryonic migratory phenotype and promote melanoma invasion through loss of E-cadherin.

Conditioned medium from irradiated keratinocytes downregulates E-cadherin in melanocytic cells. UVB irradiation induces ET-1 secretion by keratinocytes (6). We therefore determined whether E-cadherin could be downregulated in melanocytic cells by physiologic levels of ET-1 secreted by keratinocytes in response to UVB irradiation. Human keratinocytes were irradiated with 1-2 $\mathrm{mJ} / \mathrm{cm}^{2}$ of UVB, which are doses yielding less than $5 \%$ cell death in our studies. Although it is difficult to extrapolate from in vitro studies, this has been estimated as approximately half the dose that basal layer keratinocytes receive during a mild sunburn (19). Conditioned medium (UV-KCM) was then collected and used to stimulate melanocytes and melanoma cells for 40 hours. As shown (Figure 1f, left panel), UV-KCM collected from keratinocytes irradiated at $1 \mathrm{~mJ} / \mathrm{cm}^{2}$ of UVB had no effect on E-cadherin levels in melanoma cells relative to the effect of KCM collected from nonirradiated cells. In contrast, UV-KCM collected from keratinocytes irradiated at $2 \mathrm{~mJ} / \mathrm{cm}^{2}$ strongly downregulated E-cadherin approximately tenfold. Higher doses of irradiation were not used because they produced unacceptable levels of cell death (>5\%). Prior treatment of melanoma cells with BQ788 blocked the ability of UV-KCM at $2 \mathrm{~mJ} / \mathrm{cm}^{2}$ to a

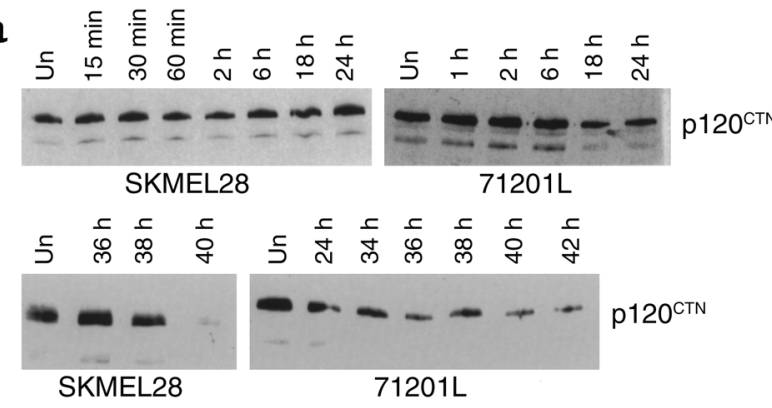

b

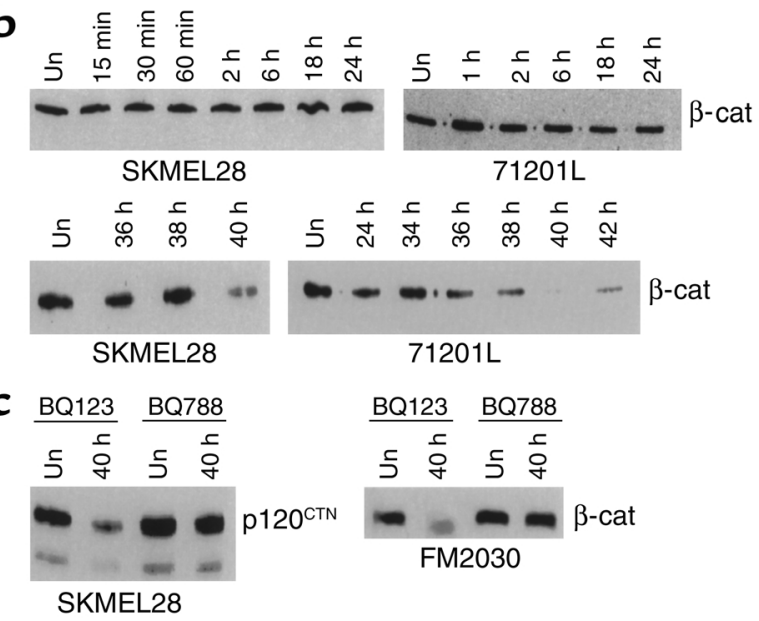

d
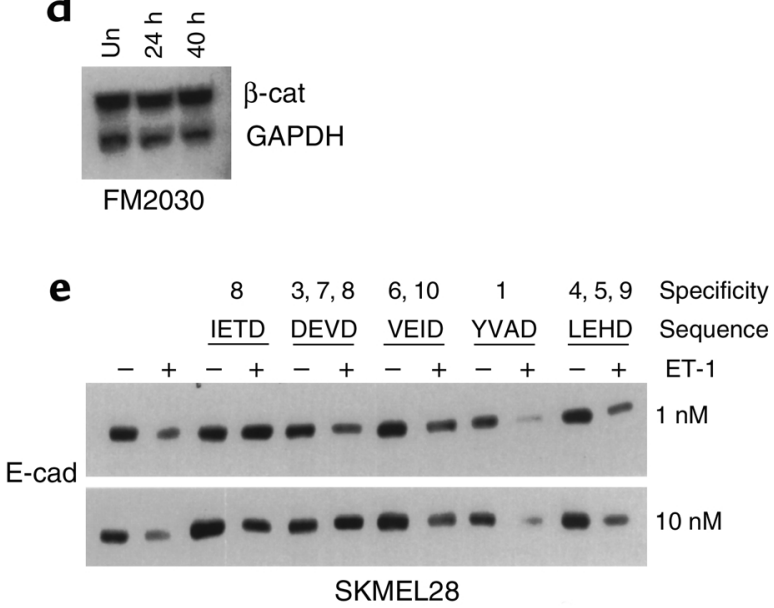

f

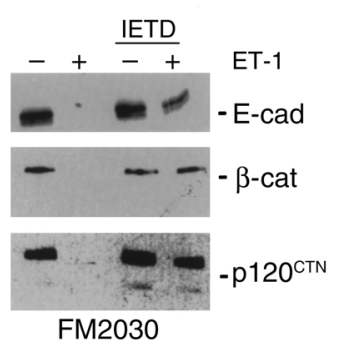

Figure 2

Downregulation of $\mathrm{p} 120^{\mathrm{CTN}}$ and $\beta$-catenin by ET-1. Lysates normalized for protein content were analyzed for p $120^{\mathrm{CTN}}$ and $\beta$-catenin protein levels were analyzed using immunoblots (internal controls for $\mathbf{a}$ and $\mathbf{b}$ are shown in Figure 1a). (a) ET-1 downregulates p120 CTN. Both large and small isoforms of p120 CTN are present. (b) ET-1 downregulates $\beta$-catenin. (c) BQ788 blocks downregulation of p120 CTN (left panel, data shown for melanoma cells) and $\beta$-catenin (right panel, data shown for melanocytes) by ET-1. (d) $\beta$-catenin Northern blot. GAPDH serves as an internal control. Identical results were obtained using melanoma cells (data not shown). (e) Cell-permeable inhibitors of caspase- 8 block downregulation of E-cadherin by ET-1. SKMEL28 cells were untreated (-) or treated (+) with 10 nM ET-1. A variety of caspase inhibitors at $1 \mathrm{nM}$ (upper panel) and $10 \mathrm{nM}$ (lower panel) concentrations were added 34 hours after ET- 1 stimulation, and cells were harvested at 40 hours. Samples were examined for E-cadherin protein levels as described previously. (f) A cell-permeable inhibitor of caspase- 8 blocks downregulation of E-cadherin, $\beta$-catenin, and p120 CTN in FM2030 cells. Identical results were obtained using melanoma cells (data not shown). Results shown are representative of at least four independent experiments. $\beta$-cat, $\beta$-catenin. 
$\mathbf{a}$

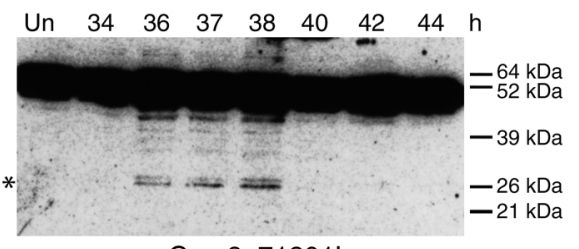

Cas-8: $71201 L$

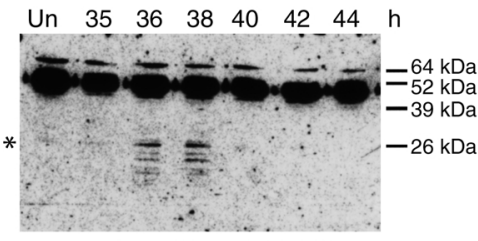

Cas-8: SKMEL28

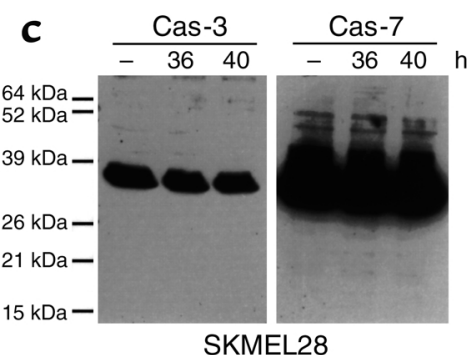

SKMEL28

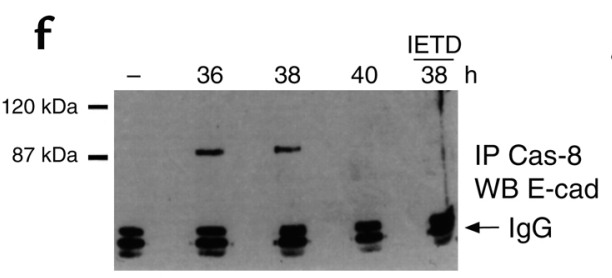

i

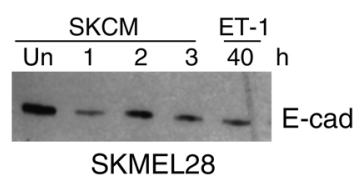

d

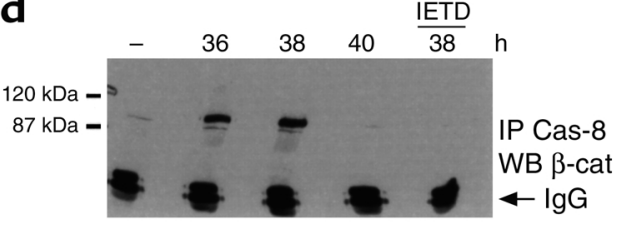

h

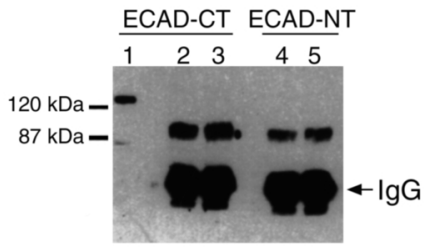

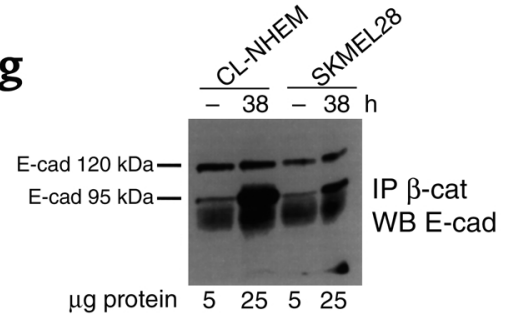

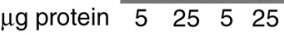

b

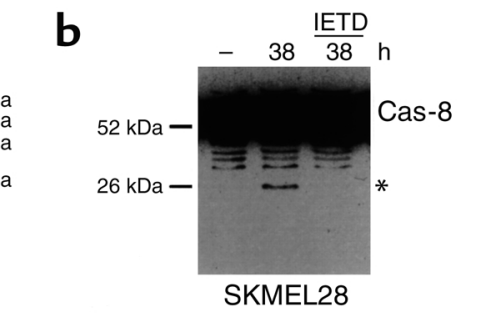

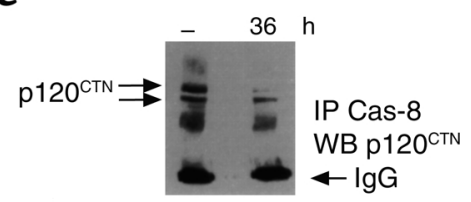

$\leftarrow \lg G$

\section{Figure 3}

ET-1 activates caspase-8. Lysates normalized for protein content were analyzed for caspase activation by immunoblot analysis. Un, unstimulated control. (a) ET-1 activates caspase-8 in melanocytes (left panel) and melanoma cells. Asterisks indicate positions of caspase-8 cleavage products. (b) IETD blocks caspase-8 activation by ET-1 in melanoma cells. Identical results were obtained using melanocytes (data not shown). (c) ET-1 does not activate caspase- 3 and caspase- 7 in melanoma cells. Expected molecular weight of caspase catalytic subunits is approximately $20 \mathrm{kDa}$. Identical results were obtained using melanocytes (data not shown). (d) Caspase-8 associates with $\beta$-catenin, (e) p120 CTN, and $(\mathbf{f})$ E-cadherin. (g) Association of $\beta$-catenin with E-cadherin in unstimulated versus ET-1-stimulated melanocytes (CL-NHEM) and melanoma cells (SKMEL28). $\beta$-catenin immune complexes were prepared from samples normalized for $\beta$-catenin content (not total protein content). (h) The 95-kDa E-cadherin fragment is detected in cell culture medium. Lane 1, full-length E-cadherin in whole cell lysates. Lanes 2-5, 95-kDa E-cadherin fragment immunoprecipitated from cell culture medium of cells treated with ET-1 for 40 hours (lanes 3 and 5) and unstimulated controls (lanes 2 and 4). Ab's directed to a large C-terminal region spanning the caspase cleavage site (ECAD-CT), as well as one directed against the extracellular domain (ECAD-NT), were used. (i) Conditioned medium collected from SKMEL28 cells (SKCM) treated for 40 hours with ET-1 (ET) and unstimulated controls (Un) was transferred to new SKMEL28 cells for the indicated times. Identical results were obtained using melanocytes (data not shown). Results shown are representative of at least four independent experiments. Cas, Caspase.

downregulate E-cadherin (Figure 1f, left panel, far right lane), demonstrating that E-cadherin downregulation by UV-KCM occurs via an $\mathrm{ET}_{\mathrm{B}}$ receptor-dependent pathway. UVB irradiation of keratinocytes induces secretion of IL- $1 \alpha$, which acts in an autocrine fashion to stimulate secretion of ET-1 (6). To determine whether IL-1 $\alpha$ stimulation of keratinocytes could substitute for UVB irradiation, we treated keratinocytes with purified recombinant human IL- $1 \alpha$ at a concentration of $40 \mathrm{pg} / \mathrm{ml}$, which corresponds to the amount of IL- $1 \alpha$ secreted by keratinocytes in response to UVB irradiation (6). IL-1KCM was collected after 4 days and used to stimulate melanocytic cells for 40 hours. IL-1-KCM also downregulated E-cadherin in melanocytic cells, and this downregulation was blocked by $\mathrm{ET}_{\mathrm{B}}$ receptor antagonist
BQ788 (Figure 1f, middle panel). In contrast, treatment of melanocytic cells with purified recombinant IL- $1 \alpha$ failed to downregulate E-cadherin (Figure 1f, right panel), providing evidence that IL- $1 \alpha$ is not the agent in UV-KCM and IL-1-KCM responsible for eliciting the response. Since keratinocytes do not secrete ET-3 (6), and since ET-1 is the only other known $\mathrm{ET}_{\mathrm{B}}$ ligand, ET- 1 is the most likely agent in UV-KCM and IL1-KCM responsible for eliciting the response.

ET- 1 concentrations in KCM were determined by ELISA. As shown in Figure 1g, levels of ET-1 secretion induced by IL- $1 \alpha$ and UVB at a dose of $2 \mathrm{~mJ} / \mathrm{cm}^{2}$ were approximately double that secreted at baseline. A UVB dose of $1 \mathrm{~mJ} / \mathrm{cm}^{2}$ did not augment ET-1 secretion by keratinocytes (Figure 1g). This suggests that 
ET-1 levels must exceed a critical threshold for E-cadherin downregulation to occur and that ET-1 levels secreted by keratinocytes at baseline are insufficient to elicit the response. UVB dose responsiveness of ET-1 secretion also correlates with ET-1 as the active agent in UVB-KCM and IL-1-KCM responsible for E-cadherin downregulation. The concentration of keratinocyte-derived ET-1 required to downregulate E-cadherin $(36 \mathrm{pM})$ is approximately 14 -fold lower than that required for the synthetic preparation of ET-1 used earlier (0.5 nM), which is likely due to a higher biologic activity. It is also possible, however, that KCM contains additional soluble factors that may augment E-cadherin downregulation by ET-1. In addition, serum albumins interfere with ET-1 receptor binding (20), and culture medium for melanocytes and melanoma cells requires serum addition for cell viability. However, keratinocyte culture medium, which is used for the UVB/IL-1 studies, is serum free and will not impair ET-1 receptor binding. Serum-free keratinocyte medium can support the growth of melanocytic cells if conditioned previously with keratinocyte factors (12).

ET-1 downregulates $p 120^{C T N}$ and $\beta$-catenin. The catenins are cytoplasmic proteins that bind E-cadherin and are critical for E-cadherin function in cellular adhesion (21). We therefore investigated the effect of ET-1 stimulation upon these proteins by analyzing their abundance every 2 hours following treatment with ET-1. In both melanocytes and melanoma cells, ET-1 stimulation decreased the protein levels of $\mathrm{p} 120^{\mathrm{CTN}}$, a member of the catenin family, markedly observed by 40 hours (Figure 2a). In SKMEL28 cells, no significant decrease was noted at early time points spanning 15 minutes to 24 hours (Figure 2a). In melanocytes, a slight (approximately twofold) decrease was noted at the 18- and 24hour time points (Figure 2a). The delayed kinetics of p120 CTN downregulation correlate well with those of E-cadherin downregulation. BQ788, a selective $\mathrm{ET}_{\mathrm{B}}$ receptor antagonist, blocked ET-1-mediated downregulation of p120 ${ }^{\mathrm{CTN}}$ levels (Figure $2 \mathrm{c}$, left panel). ET-1 also downregulated $\beta$-catenin protein in melanoma cells and melanocytes, with kinetics paralleling those observed for E-cadherin and $\mathrm{p} 120^{\mathrm{CTN}}$ downregulation (Figure $2 \mathrm{~b}$ ). No downregulation of $\beta$-catenin was observed at time points prior to 24 hours in melanoma cells. However, a slight downregulation (approximately twofold) was observed in melanocytes at the 18- and 24-hour time points, but by 34 hours, protein levels were comparable to that observed in unstimulated cells. Downregulation of $\beta$-catenin was inhibited by the selective $\mathrm{ET}_{\mathrm{B}}$ receptor antagonist $\mathrm{BQ788}$, but not by $\mathrm{BQ} 123$ (Figure 2c, right panel), again demonstrating that this response is mediated by $\mathrm{ET}_{\mathrm{B}}$. Northern blot analysis demonstrated no ET-1-dependent decrease in $\beta$-catenin mRNA abundance (Figure $2 \mathrm{~d}$ ), suggesting a posttranscriptional mechanism of downregulation. Despite the delayed onset of E-cadherin, p120 ${ }^{\mathrm{CTN}}$, and $\beta$-catenin downregulation by ET-1, the reduction in protein levels occurs rapidly during a short time period corresponding to 38-40 hours in melanoma cells and 34-40 hours in melanocytes (Figure 2, a and b), identical to that of E-cadherin downregulation. The half-life of E-cadherin is approximately 5 hours (22), and $\beta$-catenin is extremely stable when E-cadherin is expressed in cells (21). Given the stability of E-cadherin and catenin proteins, the rapidity of the observed ET-1-dependent decrease in protein levels is suggestive of a process involving the proteolytic degradation. In addition, ET- 1 induced a slight increase in the electrophoretic mobility of p $120^{\text {CTN }}$ first detected at 36-38 hours (Figure 2a) and possibly indicative of proteolytic cleavage. Since we were unable to reproduce this shift by phosphatase treatment of $\mathrm{pp} 120^{\mathrm{CTN}}$ (data not shown), we next investigated whether protease activation is involved in the downregulation of E-cadherin by ET-1.

Downregulation of E-cadherin by ET-1 is inbibited by a cellpermeable caspase-8 inbibitor. Caspases are proteases best known for their role in apoptotic cell death, although they also participate in inflammatory processes independent of apoptosis (23). During apoptosis, caspases cleave catenins and E-cadherin, resulting in a rapid decrease in protein levels (24-26). Staurosporine, a well-known activator of caspases, induces a very slight increase in the electrophoretic mobility of p120 ${ }^{\mathrm{CTN}}$, strikingly similar to the mobility shift observed in Figure $2 \mathrm{a}(27,28)$. Moreover, ET- 1 can induce apoptosis in approximately $15 \%$ of A375 melanoma cells subjected to double thymidine block, suggesting that ET-1 is capable of activating caspase family members (29). It should be noted, however, that in this study ET-1 failed to induce apoptosis in asynchronously growing cells and, in fact, can be used to support long-term proliferation of melanocytic cells (30). To determine whether caspases were involved in the downregulation of E-cadherin by ET-1, studies were carried out in the presence of cell-permeable inhibitors of different caspases (Figure 2e). Inhibitors were added to cells 34 hours after ET- 1 addition, and cells were harvested 6 hours later at the 40-hour time point. Each inhibitor was added to unstimulated cells to serve as an internal control for the effect of each inhibitor on E-cadherin levels at baseline. When compared with the appropriate internal control, only inhibitors containing the sequence IETD (specific inhibitor of caspase-8) or DEVD (inhibitor of caspase-3, -7, -8) (31) blocked downregulation of E-cadherin by ET-1. Only the caspase-8-specific inhibitor was effective at the $1 \mathrm{nM}$ concentration, which approximates the physiologic inhibitory concentration $\left(K_{\mathrm{I}}\right)$ for both inhibitors (Figure 2f, upper panel) (31). At a 10-nM concentration, both IETD and DEVD inhibitors blocked E-cadherin downregulation by ET-1 (Figure 2f, lower panel). Thus, E-cadherin downregulation is exquisitely sensitive to these inhibitors, which are routinely used in the micro- and even millimolar ranges to exert an inhibitory effect on caspase activity. Inhibitors specific for caspase-1, $-4,-5,-6,-9$, and -10 were without effect at either the $1-\mathrm{nM}$ or 
10-nM concentrations, suggesting that these caspases are not involved in the response. The caspase- 8 inhibitor (IETD) also blocked downregulation of $\beta$-catenin and $\mathrm{p} 120^{\mathrm{CTN}}$ by ET-1 (Figure 2f). These results suggest that caspase-8, and possibly caspase- 3 and -7 , are activated in response to ET- 1 stimulation and participate in the downregulation of E-cadherin, $\beta$-catenin, and $\mathrm{p} 120^{\mathrm{CTN}}$ proteins by ET- 1 .

ET-1 activates caspase-8 but fails to activate executioner caspase-3 and -7. Immunoblot analysis was performed to determine whether caspase- $8,-3$, or -7 were activated in response to ET-1 stimulation of melanocytic cells. Of these three caspases, only caspase- 8 was activated in ET-1-stimulated cells, as evidenced by the proteolytic processing of its 54-kDa zymogen (Figure 3a, left and right panels). Caspase- 8 activation was monitored at 2-hour intervals and is first detectable approximately 36 hours after ET-1 stimulation (early time points not shown), thus correlates very well with the delayed downregulation of E-cadherin and catenin proteins by ET-1. The cell-permeable inhibitor of caspase-8, shown in Figure 2 to inhibit E-cadherin downregulation, also blocked the proteolytic processing of caspase- 8 (Figure $3 \mathrm{~b}$ ), indicating that caspase- 8 activation may be required for downregulation of E-cadherin by ET-1. No proteolytic processing of caspase- 3 or -7 was detectable in ET-1-treated cells (Figure 3c). Observation of cells for cell viability was conducted and, as reported in the literature (30), no decrease in cell viability was noted as far out as 72 hours after ET-1 stimulation. The apparent failure of ET-1 to activate distal caspases in melanocytic cells, despite the activation of caspase- 8 , is consistent with its failure to induce apoptosis in these cells. It is possible that the low level of caspase- 8 activation observed in these studies is sufficient to downregulate E-cadherin and catenin proteins, but insufficient to activate distal executioner caspases. Furthermore, it is well established that ET-1, at the concentrations used in this study, does not induce cell death in either melanocytes or melanoma cells $(4,5,12)$. In addition, melanoma cells and nevus cells express members of the IAP (inhibitor of apoptosis protein) family, including survivin and livin $(32,33)$. IAPs prevent activation of caspase- 3 and -7 , while they have no effect on caspase- 8 activation (32). These data therefore demonstrate that activation of caspase- 8 is involved in the response of melanocytic cells to ET-1, which is associated with downregulation of E-cadherin and catenin proteins. While other caspases might also be involved, it is clear that caspase- 3 and -7 do not play a role in the ET-1-mediated response, and their inactivity likely prevents the induction of apoptosis by ET-1 in these cells.

ET-1 induces a transient association between caspase-8, $E$-cadherin, and $\beta$-catenin. Immunoprecipitation studies were performed to determine whether ET-1 induces an association between caspase-8, E-cadherin, and catenin proteins. In unstimulated cells, caspase- 8 does not associate with $\beta$-catenin (Figure $3 \mathrm{~d}$ ). However, treatment of cells with ET-1 induces a transient association of $\beta$-catenin with caspase- 8 , which was strongly detected at the 36- and 38-hour time points as shown, but that is not observed at 40 hours. These time points coincide with the kinetics of caspase- 8 activation and correlate well with the onset of E-cadherin and catenin downregulation by ET-1 shown earlier. No association was detected prior to the 36-hour time point (data not shown). Caspase-8: $\beta$-catenin association is specifically inhibited in vivo by treatment of cells with a cell-permeable caspase-8 inhibitor (Figure $3 \mathrm{~d}$, far right lane). Since this inhibitor also blocks downregulation of $\beta$-catenin, these results suggest that the transient association with caspase-8 plays a role in downregulation of $\beta$-catenin by ET-1. In addition, since this inhibitor prevents caspase- 8 activation, these data further suggest that $\beta$-catenin associates predominantly with the activated form of caspase- 8 . In contrast, $\mathrm{p} 120^{\mathrm{CTN}}$ was associated with caspase- 8 in both ET-1-stimulated cells and in unstimulated controls, although to a lesser extent, suggesting that a subpopulation of p $120^{\mathrm{CTN}}$ might form complexes exclusive of E-cadherin and $\beta$-catenin (Figure 3e). ET-1 also induces a transient association between caspase- 8 and E-cadherin at 36 and 38 hours (Figure 3f), which can be blocked in vivo by a cell-permeable inhibitor of caspase-8 (Figure 3f, far right lane). Time points prior to 36 hours failed to reveal caspase8:E-cadherin association (data not shown). As for $\beta$-catenin, these data suggest that transient association with caspase-8 plays a role in E-cadherin downregulation by ET- 1 and that E-cadherin associates with the activated form of caspase-8. Interestingly, the apparent molecular weight of E-cadherin detected in association with caspase- 8 is approximately $95 \mathrm{kDa}, 25 \mathrm{kDa}$ smaller than the expected molecular weight. This $95-\mathrm{kDa}$ fragment can also be detected in $\beta$-catenin immune complexes from ET-1-treated melanocytic cells but not in unstimulated controls (Figure $3 \mathrm{~g}$ ). In contrast with caspase- 8 immune complexes, however, $\beta$-catenin immune complexes are additionally associated with full-length E-cadherin. A caspase cleavage site has been identified at amino acid site 750 of human E-cadherin, and the molecular weight of the large fragment generated by cleavage at this site is $94 \mathrm{kDa}$ (26). During apoptosis, the $94-\mathrm{kDa}$ E-cadherin subfragment is shed from the cell surface and is therefore undetectable in whole cell lysates (26). Although we are able to detect this E-cadherin fragment in caspase- 8 and $\beta$-catenin immune complexes, as expected, it cannot be detected in whole cell lysates (Figure 1a). We can, however, detect a $95-\mathrm{kDa}$ E-cadherin fragment in culture medium collected from cells, but since its levels are not significantly increased by ET-1 treatment, it is likely that it is also shed during normal turnover of E-cadherin (Figure $3 \mathrm{~h}$ ).

Overall, these results demonstrate that caspase- 8 associates with the E-cadherin: $\beta$-catenin complex in an ET-1-dependent manner. While not proven, it is reasonable to conclude that this association likely plays a role in downregulation of E-cadherin and $\beta$-catenin by ET-1. The mechanism by which ET-1 downregulates E-cadherin and catenin proteins has been described 
previously in the literature $(24,26)$. Briefly outlined, caspase activation results in the cleavage of E-cadherin, with shedding of a large amino-terminal fragment and release of the cytoplasmic catenin-binding domain. Since E-cadherin stabilizes catenin proteins at the plasma membrane (21), cleavage of E-cadherin results in destabilization and rapid degradation of catenin proteins by targeting to the proteosome (34). The pathway we describe here is novel in that it is induced by a physiologic agent (ET-1) and caspase activation occurs independently of apoptosis. We also describe here, we believe for the first time, a direct association of caspase- 8 with the E-cadherin:catenin complex in vivo, which is consistent with the proposed mechanism of action. A curious feature of ET- 1 action, which is not understood for any of its reported activities, is the delayed response. Studies are ongoing to understand the molecular basis for the extensive delay between ET-1 treatment of cells and its physiological effects. It is possible that ET-1 induces the secretion of a factor that acts upon melanocytic cells in an autocrine fashion to downregulate E-cadherin and induce delayed morphology changes, as reported in other studies $(11,12)$. It may be that this factor needs to accumulate in culture medium and reach a critical concentration to mediate its effects. In support of this hypothesis, conditioned medium collected from melanocytic cells after 40 hours of ET- 1 stimulation rapidly downregulates E-cadherin when transferred to new cells, while conditioned medium from unstimulated controls does not (Figure 3i). Conditioned medium from ET-1-stimulated cells downregulates E-cadherin within 1 hour to levels comparable to that seen after 40 hours of stimulation with purified ET-1. These data are consistent with the notion that ET-1 stimulates the secretion of a factor that acts in an autocrine fashion to downregulate E-cadherin in melanocytic cells. It likely takes 40 hours for concentrations of this factor to be effective. Future studies will be aimed at the identification of this factor and at the elucidation of its activities.

\section{Discussion}

We have demonstrated that ET-1 downregulates E-cadherin through a novel mechanism involving caspase-8 activation that occurs in the absence of apoptotic cell death. One of the early hallmarks of apoptosis is cytoskeletal reorganization and retraction from neighboring cells, possibly due to E-cadherin downregulation (25). Our study suggests that while this process requires caspase activation, it can be uncoupled from apoptotic cell death. We also have demonstrated that caspase- 8 can associate, in an inducible fashion, with members of the E-cadherin:catenin complex, which may decrease the stability of this complex, leading to a rapid decrease in protein levels. Our results therefore suggest that caspases, in addition to mediating apoptotic events, also participate in processes affecting adhesion and cell phenotype.
The keratinocyte plays an important role in regulating normal functions of the melanocyte in the epidermis, including growth rate and pigment production (35). Thus, aberrant signaling from the keratinocyte may contribute to the abnormal growth patterns of melanocytic cells observed during melanoma development and progression. While ET-1 secreted at low levels plays a role in maintaining melanocyte viability and in stimulating melanin production in response to UVR (6), epidermal ET-1 hypersecretion, coupled with the increase in $\mathrm{ET}_{\mathrm{B}}$ receptor expression that occurs in melanoma progression, may generate local populations of melanocytes with the potential to escape the epidermis due to a loss of E-cadherin. During the initial phases of melanoma progression, such populations may clinically resemble nevi (moles), which are known to express decreased E-cadherin levels and can demonstrate invasion into the dermis $(36,37)$. During later stages of melanoma development, such populations may represent invasive melanomas. There are two possible mechanisms that might account for focal epidermal ET-1 hypersecretion: (a) chronic UV damage to the epidermis may generate focal populations of keratinocytes secreting abnormally high levels of ET-1, as has been demonstrated in skin samples from lentigo senilis lesions (8), and (b) alternatively, factors produced by neoplastic melanocytic cells themselves may act upon keratinocytes in a paracrine fashion to stimulate constitutive ET-1 hypersecretion. Cells from nevi and thin melanomas secrete low levels of TNF- $\alpha$, while thick and metastatic melanomas additionally secrete IL- $1 \alpha$ (38). Both TNF- $\alpha$ and IL- $1 \alpha$ are potent inducers of ET-1 secretion in the keratinocyte $(7,38)$. Thus TNF- $\alpha$ and/or IL- $1 \alpha$ secretion by atypical melanocytes may create an abnormal signaling loop resulting in constitutive ET-1 secretion by adjacent keratinocytes. While exposure to high levels of ET-1 may promote a proliferative, migratory, and invasive state in melanocytic cells, possibly inducing mole formation and lentigines, such exposure is unlikely to be sufficient for the induction of frank malignancy. Acquired or germline mutations of genes that affect growth-regulatory pathways are required for melanocytic tumors to be malignant. However, activation of the ET- $1 / \mathrm{ET}_{\mathrm{B}}$ pathway, in combination with these mutations, may generate melanomas with the potential for local invasion into dermal regions through downregulation of E-cadherin. It is interesting that studies have suggested that Ras activation is required for melanoma invasiveness (39). While activating mutations of Ras are rarely found in malignant melanoma, ET- 1 is a known activator of Ras (40) and may therefore play a complex role in invasiveness. Melanocytic cells that have escaped the epidermis and invaded the underlying dermis, moreover, might continue to be stimulated by ET- 1 of endothelial cell origin. High levels of endothelial ET-1 are localized to dermal perivascular regions (5). Since ET-1 is chemotactic for melanoma cells, it may recruit melanoma cells to the vasculature, thereby promoting metastatic spread (5). 
ET- 1 is secreted by a variety of cancer cells, including prostate, mammary, pancreatic, colon, and ovarian cancer cells, and stimulates their proliferation in an autocrine fashion $(5,41,42)$. In clinical trials, systemic delivery of ET receptor antagonists is showing promise in the treatment of metastatic prostate cancer (43). In view of our findings, ET-1, through downregulation of E-cadherin, might participate with other factors in promoting growth and invasiveness of carcinomas.

\section{Acknowledgments}

This work was supported by grants from The Robert Wood Johnson Foundation (S. Jamal), UNCF/MERCK Science Initiative (S. Jamal), and the NIH (R.J. Schneider). We are grateful to I.M. Freedberg, J. Kiatjewski, G. Laroia, and E.B. Ziff for their careful reading of the manuscript, and to Micki Blumenberg for help with keratinocyte irradiations.

1. Tang, A., et al. 1994. E-cadherin is the major mediator of human melanocyte adhesion to keratinocytes in vitro. J. Cell Sci. 107:983-992.

2. Hsu, M.Y., et al. 2000. E-cadherin expression in melanoma cells restores keratinocyte-mediated growth control and down-regulates expression of invasion-related adhesion receptors. Am. J. Pathol. 156:1515-1525.

3. Valyi-Nagy, I.T., et al. 1993. Undifferentiated keratinocytes control growth, morphology, and antigen expression of normal melanocytes through cell-cell contact. Lab Invest. 69:152-159.

4. Imokawa, G., Yada, Y., and Kimura, M. 1996. Signaling mechanisms of endothelin-induced mitogenesis and melanogenesis in human melanocytes. Biochem. J. 314:305-312.

5. Yohn, J.J., et al. 1994. Human melanoma cells express functional endothelin-1 receptors. Biochem. Biophys. Res. Commun. 201:449-457.

6. Imokawa, G., Yada, Y., and Miyagishi, M. 1992. Endothelins secreted from human keratinocytes are intrinsic mitogens for human melanocytes. J. Biol. Chem. 267:24675-24680.

7. Ahn, G.Y., et al. 1998. The expression of endothelin-1 and its binding sites in mouse skin increased after ultraviolet B irradiation or local injection of tumor necrosis factor alpha. J. Dermatol. 25:78-84

8. Kadono, S., Manaka, I., Kawashima, M., Kobayashi, T., and Imokawa, G. 2001. The role of epidermal endothelin cascade in the hyperpigmentation mechanism of lentigo senilis. J. Invest. Dermatol. 114:571-577.

9. Demunter, A., De Wolf-Peeters, C., Degreef, C., Stas, M., and van den Oord, J.J. 2001. Expression of the endothelin-B receptor in pigment cell lesions of the skin. Evidence for its role as tumor progression marker in malignant melanoma. Virchows Arch. 438:485-491.

10. Lahav, R., Heffner, M., and Patterson, P.H. 1999. An endothelin receptor $\mathrm{B}$ antagonist inhibits growth and induces cell death in human melanoma cells in vitro and in vivo. Proc. Natl. Acad. Sci. USA. 96:11496-11500.

11. Scott, G., Cassidy, L., and Abdel-Malek, Z. 1997. Alpha-melanocyte-stimulating hormone and endothelin-1 have opposing effects on melanocyte adhesion, migration, and pp125FAK phosphorylation. Exp. Cell Res. 237:19-28.

12. Hara, M., Yaar, M., and Gilchrest, B.A. 1995. Endothelin-1 of keratinocyte origin is a mediator of melanocyte dendricity. J. Invest. Dermatol. 105:744-748.

13. Marsault, R., Vigne, P., and Frelin, C. 1991. The irreversibility of endothelin action is a property of a late intracellular signalling event. Biochem. Biophys. Res. Commun. 179:1408-1413.

14. Baynash, A.G., et al. 1994. Interaction of endothelin-3 with endothelin$B$ receptor is essential for development of epidermal melanocytes and enteric neurons. Cell. 79:1277-1285.

15. Eberle, J. 1999. Downregulation of endothelin B receptor in human melanoma cell lines parallel to differentiation genes. J. Invest. Dermatol. 112:925-933.

16. Kang, H.Y., Kang, W.H., and Lee, C. 1998. Endothelin-B receptor-mediated $\mathrm{Ca} 2+$ signaling in human melanocytes. Pflugers Arch. 435:350-356.

17. Huggins, J.P., and Pelton, J.T. 1997. Endothelins in biology and medicine. CRC Press Inc. Boca Raton, Florida, USA. 13-15.
18. Reid, K., et al. 1996. Multiple roles for endothelin in melanocyte development: regulation of progenitor number and stimulation of differentiation. Development. 122:3911-3919.

19. Moehrle, M., Koehle, W., Dietz, K., and Lischka, G. 2000. Reduction of minimal erythema dose by sweating. Photodermatol. Photoimmunol. Photomed. 16:260-262.

20. Wu-Wong, J.R., et al. 1996. Endothelins and endothelin receptor antagonists: binding to plasma proteins. Life Sci. 58:1839-1847.

21. Papkoff, J. 1997. Regulation of complexed and free catenin pools by distinct mechanisms. Differential effects of Wnt-1 and v-Src. J. Biol. Chem. 272:4536-4543.

22. Stewart, D.B., Barth, A.I., and Nelson, W.J. 2000. Differential regulation of endogenous cadherin expression in Madin-Darby canine kidney cells by cell-cell adhesion and activation of beta-catenin signaling. J. Biol. Chem. 275:20707-20716.

23. Thornberry, N.A., and Lazebnik, Y. 1998. Caspases: enemies within. Science. 281:1312-1316.

24. Herren, B., Levkau, B., Raines, E.W., and Ross, R. 1998. Cleavage of betacatenin and plakoglobin and shedding of VE-cadherin during endothelial apoptosis: evidence for a role for caspases and metalloproteinases. Mol. Biol. Cell. 9:1589-1601.

25. Brancolini, C., Lazarevic, D., Rodriguez, J., and Schneider, C. 1997. Dismantling cell-cell contacts during apoptosis is coupled to a caspasedependent proteolytic cleavage of beta-catenin. J. Cell Biol. 139:759-771.

26. Steinhusen, U., et al. 2001. Cleavage and shedding of E-cadherin after induction of apoptosis. J. Biol. Chem. 276:4972-4980.

27. Aono, S., Nakagawa, S., Reynolds, A.B., and Takeichi, M. 1999. p120(ctn) acts as an inhibitory regulator of cadherin function in colon carcinoma cells. J. Cell Biol. 145:551-562.

28. Ohkubo, T., and Ozawa, M. 1999. p120(ctn) binds to the membraneproximal region of the E-cadherin cytoplasmic domain and is involved in modulation of adhesion activity. J. Biol. Chem. 274:21409-21415.

29. Okazawa, M., Shiraki, T., Ninomiya, H., Kobayashi, S., and Masaki, T. 1998. Endothelin-induced apoptosis of A375 human melanoma cells. J. Biol. Chem. 273:12584-12592.

30. Swope, V.B., Medrano, E.E., Smalara, D., and Abdel-Malek, Z.A. 1995. Long-term proliferation of human melanocytes is supported by the physiologic mitogens alpha-melanotropin, endothelin-1, and basic fibroblast growth factor. Exp. Cell Res. 217:453-459.

31. Garcia-Calvo, M., et al. 1998. Inhibition of human caspases by peptidebased and macromolecular inhibitors. J. Biol. Chem. 273:32608-32613.

32. Kasof, G.M., and Gomes, B.C. 2000. Livin, a novel inhibitor of apoptosis protein family member. J. Biol. Chem. 276:3238-3246.

33. Chiodino, C., et al. 1999. Communication: expression of the novel inhibitor of apoptosis survivin in normal and neoplastic skin. J. Invest. Dermatol. 113:415-418.

34. Orford, K., Crockett, C., Jensen, J.P., Weissman, A.M., and Byers, S.W. 1997. Serine phosphorylation-regulated ubiquitination and degradation of beta-catenin. J. Biol. Chem. 272:24735-24738.

35. Shih, I.M., Elder, D.E., Hsu, M.Y., and Herlyn, M. 1994. Regulation of Mel-CAM/MUC18 expression on melanocytes of different stages of tumor progression by normal keratinocytes. Am. J. Pathol. 145:837-845.

36. Silye, R., et al. 1998. E-cadherin/catenin complex in benign and malignant melanocytic lesions. J. Pathol. 186:350-355.

37. Danen, E.H., et al. 1996. E-cadherin expression in human melanoma. Melanoma Res. 6:127-131.

38. Moretti, S., et al. 1999. Immunohistochemical evidence of cytokine networks during progression of human melanocytic lesions. Int. J. Cancer. 84:160-168.

39. Chin, L., et al. 1997. Cooperative effects of INK4a and ras in melanoma susceptibility in vivo. Genes Dev. 11:2822-2834.

40. Herman, W.H., and Simonson, M.S. 1995. Nuclear signaling by endothelin-1. A Ras pathway for activation of the c-fos serum response element. J. Biol. Chem. 270:11654-11661.

41. Nelson, J.B., et al. 1996. Endothelin-1 production and decreased endothelin B receptor expression in advanced prostate cancer. Cancer Res. 56:663-668.

42. Bagnato, A., Tecce, R., Di Castro, V., and Catt, K.J. 1997. Activation of mitogenic signaling by endothelin 1 in ovarian carcinoma cells. Cancer Res. 57:1306-1311.

43. Carducci, M.A., Nelson, J.B., Padley, R.J., Janus, T., and Hippensteel, R. 2001. The Endothelin-A receptor antagonist atrasentan (ABT-627) delays clinical progression in hormone refractory prostate cancer: a multinational, randomized, double blind, placebo controlled trial. Proceedings of the American Society of Clinical Oncology Program 2001. San Francisco, California, USA. 694. (Abstr.) 